\title{
Front Matter: Volume 7183
}

, "Front Matter: Volume 7183," Proc. SPIE 7183, Multiphoton Microscopy in the Biomedical Sciences IX, 718301 (16 March 2009); doi: 10.1117/12.824924 SPIE. Event: SPIE BiOS, 2009, San Jose, California, United States 


\title{
Multiphoton Microscopy in the Biomedical Sciences IX
}

\author{
Ammasi Periasamy \\ Peter T. C. So \\ Editors
}

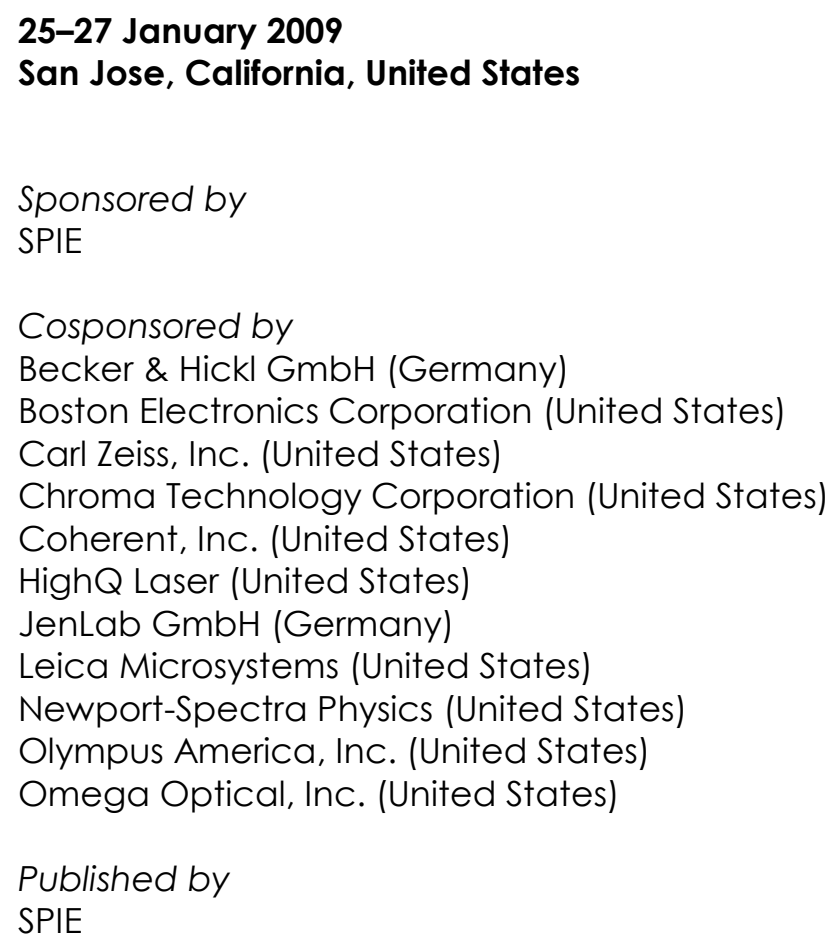

Volume 7183 
The papers included in this volume were part of the technical conference cited on the cover and title page. Papers were selected and subject to review by the editors and conference program committee. Some conference presentations may not be available for publication. The papers published in these proceedings reflect the work and thoughts of the authors and are published herein as submitted. The publisher is not responsible for the validity of the information or for any outcomes resulting from reliance thereon.

Please use the following format to cite material from this book:

Author(s), "Title of Paper," in Multiphoton Microscopy in the Biomedical Sciences IX, edited by Ammasi Periasamy, Peter T. C. So, Proceedings of SPIE Vol. 7183 (SPIE, Bellingham, WA, 2009) Article CID Number.

ISSN 1605-7422

ISBN 9780819474292

Published by

SPIE

P.O. Box 10, Bellingham, Washington 98227-0010 USA

Telephone +1 3606763290 (Pacific Time) · Fax +1 3606471445

SPIE.org

Copyright (C) 2009, Society of Photo-Optical Instrumentation Engineers.

Copying of material in this book for internal or personal use, or for the internal or personal use of specific clients, beyond the fair use provisions granted by the U.S. Copyright Law is authorized by SPIE subject to payment of copying fees. The Transactional Reporting Service base fee for this volume is $\$ 18.00$ per article (or portion thereof), which should be paid directly to the Copyright Clearance Center (CCC), 222 Rosewood Drive, Danvers, MA 01923. Payment may also be made electronically through CCC Online at copyright.com. Other copying for republication, resale, advertising or promotion, or any form of systematic or multiple reproduction of any material in this book is prohibited except with permission in writing from the publisher. The CCC fee code is 1605 $7422 / 09 / \$ 18.00$.

Printed in the United States of America.

Publication of record for individual papers is online in the SPIE Digital Library.

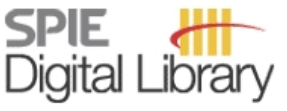

SPIEDigitallibrary.org

Paper Numbering: Proceedings of SPIE follow an e-First publication model, with papers published first online and then in print and on CD-ROM. Papers are published as they are submitted and meet publication criteria. A unique, consistent, permanent citation identifier (CID) number is assigned to each article at the time of the first publication. Utilization of CIDs allows articles to be fully citable as soon they are published online, and connects the same identifier to all online, print, and electronic versions of the publication. SPIE uses a six-digit CID article numbering system in which:

- The first four digits correspond to the SPIE volume number.

- The last two digits indicate publication order within the volume using a Base 36 numbering system employing both numerals and letters. These two-number sets start with $00,01,02,03,04$, $05,06,07,08,09,0 A, 0 B \ldots$. 0Z, followed by 10-1Z, 20-2Z, etc.

The CID number appears on each page of the manuscript. The complete citation is used on the first page, and an abbreviated version on subsequent pages. Numbers in the index correspond to the last two digits of the six-digit CID number. 


\section{Contents}

\section{xi Conference Committee}

xiii $\quad$ Abstract only: STED microscopy made simple (Best Student Poster Award) [7183-77]

A. Schönle, L. Kastrup, K. Willig, D. Wildanger, B. Harke, B. Rankin, C. Eggeling, A. Egner, S. W. Hell, Max-Planck-Institut für Biophysikalische Chemie (Germany)

xiv Abstract only: Optimization of fluorescence collection in multiphoton microscopy (Best Student Poster Award) [7183-91]

J. Zinter, M. J. Levene, Yale Univ. (United States)

\section{SESSION 1 KEYNOTE SESSION}

718302 What is behind all those lifetimes anyway, and where do we go from here? (Keynote Paper) [7183-01]

Y.-C. Chen, B. Q. Spring, C. Buranachai, Univ. of Illinois at Urbana-Champaign (United States); G. Malachowski, Bioscience Applications, Pty. Ltd. (Australia); R. M. Clegg, Univ. of Illinois at Urbana-Champaign (United States)

718303 Multicolor fluctuation spectroscopy in cells (Keynote Paper) [7183-02]

M. A. Digman, E. Gratton, Univ. of California, Irvine (United States)

\section{SESSION 2 FLIM/FRET/FCS I}

718305 Fast-acquisition multispectral FLIM by parallel TCSPC [7183-04]

W. Becker, B. Su, A. Bergmann, Becker \& Hickl GmbH (Germany)

718306 The characterization of optimized fluorescent proteins for Förster resonance energy transfer microscopy (Invited Paper) [7183-05]

R. N. Day, Univ. of Virginia Health System (United States); Y. Sun, Univ. of Virginia (United States); C. F. Booker, S. Kumari, Univ. of Virginia Health System (United States); A. Periasamy, Univ. of Virginia (United States); M. Davidson, Florida State Univ. (United States)

718308 Adding new dimensions to fluorescence microscopy [7183-07] C. Biskup, J. Kusch, Friedrich-Schiller-Univ. Jena (Germany); E. Schulz, Fachhochschule Schmalkalden (Germany); B. Hoffmann, V. Nache, Friedrich-Schiller-Univ. Jena (Germany); F. Schwede, Biolog Life Science Institute (Germany); F. Lehmann, Dyomics GmbH (Germany); K. Benndorf, Friedrich-Schiller-Univ. Jena (Germany)

718309 Metabolic mapping of cell culture growth by NADH fluorescence lifetime imaging [7183-08] V. Ghukasyan, T. Buryakina, F.-J. Kao, National Yang-Ming Univ. (Taiwan) 
7183 OA SLIM: multispectral FLIM with wide applications in cell biology (Invited Paper) [7183-09] A. Rück, F. Dolp, Institute for Laser Technologies in Medicine and Metrology (Germany); C. Steinmetz, B. von Einem, C. A. F. von Arnim, Univ. Ulm (Germany)

$71830 \mathrm{C}$ New multichannel photon timing instrumentation with independent synchronized channels and high count rate for FLIM and correlation analysis [7183-11]

M. Wahl, PicoQuant GmbH (Germany); G. Kell, Fachhochschule Brandenburg (Germany); P. Kapusta, H.-J. Rahn, T. Roehlicke, R. Erdmann, PicoQuant GmbH (Germany)

7183 OD Filtered FCS and species cross correlation function [7183-12]

S. Felekyan, S. Kalinin, A. Valeri, C. A. M. Seidel, Heinrich-Heine-Univ. Düsseldorf (Germany)

\section{SESSION 4 FLIM/FRET/FCS III}

7183 0l Angular domain fluorescent lifetime imaging in turbid media [7183-17]

F. Vasefi, Simon Fraser Univ. (Canada) and Lawson Health Research Institute (Canada);

E. Ng, Lawson Health Research Institute (Canada) and Univ. of Western Ontario (Canada);

B. Kaminska, G. H. Chapman, Simon Fraser Univ. (Canada); J. J. L. Carson, Lawson Health

Research Institute (Canada) and Univ. of Western Ontario (Canada)

$71830 \mathrm{~J} \quad$ Voltage sensitive dyes used for FLIM studied by two-photon-excitation [7183-18]

T. Gensch, Research Ctr. Jülich (Germany)

7183 OK Analysis of NAD(P)H fluorescence components in cardiac myocytes from human biopsies: a new tool to improve diagnostics of rejection of transplanted patients [7183-19]

Y. Cheng, CHU Sainte-Justine (Canada); A. Mateasik, International Laser Ctr. (Slovakia);

N. Poirier, J. Miró, N. Dahdah, CHU Sainte-Justine (Canada) and Univ. of Montreal (Canada);

D. Chorvat, Jr., International Laser Ctr. (Slovakia); A. Chorvatova, CHU Sainte-Justine

(Canada) and Univ. of Montreal (Canada)

7183 OL Deep-tissue multiphoton fluorescence lifetime microscopy for intravital imaging of protein-protein interactions [7183-117]

G. O. Fruhwirth, D. R. Matthews, A. Brock, M. Keppler, King's College London (United Kingdom); B. Vojnovic, King's College London (United Kingdom) and Univ. of Oxford (United Kingdom); T. Ng, S. Ameer-Beg, King's College London (United Kingdom)

\section{SESSION 5 CARS MICROSCOPY I}

$71830 Q \quad$ Optimizing CARS signal using coherent control methods [7183-25]

V. S. Malinovsky, MagiQ Technologies, Inc. (United States) 
7183 OR Multi-focus CARS microscopy using microlens array scanner for realtime molecular spectral imaging [7183-27]

M. Hashimoto, T. Minamikawa, T. Araki, K. Fujita, Osaka Univ. (Japan); S. Kawata, Osaka Univ. (Japan) and RIKEN (Japan)

7183 OT CARS microscopy using linearly chirped ultrafast laser pulses [7183-29]

I. Rocha-Mendoza, W. Langbein, P. Borri, Cardiff Univ. (United Kingdom)

7183 OU CARS and SHG microscopy for the characterization of bacterial cellulose (Invited Paper) [7183-30]

A. Enejder, C. Brackmann, A. Bodin, M. Åkeson, P. Gatenholm, Chalmers Univ. of Technology (Sweden)

\section{SESSION 7 CARS MICROSCOPY III}

7183 OV Enhancing two-color absorption, self-phase modulation, and Raman microscopy signatures in tissue with femtosecond laser pulse shaping (Invited Paper) [7183-31]

M. C. Fischer, I. Piletic, Duke Univ. (United States); D. Fu, Princeton Univ. (United States);

T. E. Matthews, Duke Univ. (United States); H. Liu, Princeton Univ. (United States); P. Samineni, B. Li, W. S. Warren, Duke Univ. (United States)

7183 OW Ultrafast multiphoton microscopy with high-order spectral phase distortion compensation (Invited Paper) [7183-32]

Y. Andegeko, D. Pestov, V. V. Lozovoy, M. Dantus, Michigan State Univ. (United States)

7183 OX Spectral phase shaping for high resolution CARS spectroscopy around $3000 \mathrm{~cm}^{-1}$ [7183-33]

A. C. W. van Rhijn, S. Postma, J. P. Korterik, J. L. Herek, H. L. Offerhaus, Univ. Twente (Netherlands)

7183 OY Pulse shaping for background free broadband CARS [7183-34]

Y. J. Lee, M. T. Cicerone, National Institute of Standards and Technology (United States)

$7183 \mathrm{OZ}$ High performance multimodal CARS microscopy using a single femtosecond source [7183-35]

A. F. Pegoraro, National Research Council Canada (Canada) and Queen's Univ. (Canada);

A. Ridsdale, D. J. Moffatt, J. P. Pezacki, National Research Council Canada (Canada);

A. Stolow, National Research Council Canada (Canada) and Queen's Univ. (Canada)

718310 Background free CARS imaging by local phase detection [7183-36]

M. Jurna, J. P. Korterik, C. Otto, L. Herek, H. L. Offerhaus, Univ. Twente (Netherlands)

718311 Optical pulse shaping for selective excitation of coherent molecular vibrations by stimulated Raman scattering [7183-37]

J. B. Geddes III, D. L. Marks, S. A. Boppart, Univ. of Illinois at Urbana-Champaign (United States) 
718312 Coherent anti-Stokes generation from single nanostructures (Invited Paper) [7183-38] H. Kim, T. Sheps, D. K. Taggart, P. G. Collins, R. M. Penner, E. O. Potma, Univ. of California, Irvine (United States)

$718314 \quad$ A new light source for multimodal multiphoton microscopy including CARS [7183-39] I. Rimke, APE GmbH (Germany); V. Siffrin, R. Niesner, T. Levenberger, F. Zipp, Univ. Berlin (Germany); E. Büttner, G. Stibenz, APE GmbH (Germany)

718315 Coupling CARS with multiphoton fluorescence and high harmonic generation imaging modalities using a femtosecond laser source [7183-40]

H. Chen, M. N. Slipchenko, J. Zhu, K. K. Buhman, J.-X. Cheng, Purdue Univ. (United States)

718316 Analytical capabilities of nonlinear Raman microspectroscopic imaging [7183-41]

R. Arora, G. I. Petrov, V. V. Yakovlev, Univ. of Wisconsin, Milwaukee (United States)

718318 Fiber lasers for CARS microscopy (Invited Paper) [7183-24]

F. W. Wise, K. Kieu, Cornell Univ. (United States); B. Saar, G. Holtom, S. Xie, Harvard Univ. (United States)

\section{SESSION 9 TECHNOLOGY DEVELOPMENT AND APPLICATIONS}

718319 Clinical multiphoton tomography and clinical two-photon microendoscopy (Invited Paper) [7183-44]

K. König, Saarland Univ. (Germany) and JenLab GmbH (Germany); R. Bückle, M. Weinigel, JenLab GmbH (Germany); P. Elsner, M. Kaatz, Friedrich-Schiller-Univ. Jena (Germany)

$71831 \mathrm{~A}$ Two-photon imaging and nanoprocessing of stem cells with sub-20 fs laser pulses [7183-45] A. Uchugonova, Fraunhofer-Institut für Biomedizinische Technik (Germany) and Saarland Univ. (Germany); A. Isemann, Femtolasers Produktions GmbH (Austria); R. Bückle, JenLab GmbH (Germany); W. Watanabe, National Institute of Advanced Industrial Science and Technology (Japan); K. König, Saarland Univ. (Germany) and JenLab GmbH (Germany)

7183 1B Three-dimensional (3D) high-speed imaging and fabrication system based on ultrafast optical pulse manipulation [7183-46]

D. Kim, P. T. C. So, Massachusetts Institute of Technology (United States)

7183 1J Adaptive optics for multiphoton microscopy [7183-49]

D. Débarre, T. Wilson, M. J. Booth, Univ. of Oxford (United Kingdom)

$71831 \mathrm{~K} \quad$ Multiphoton microscopy by multiexcitonic ladder climbing in colloidal quantum dots [7183-50]

N. R. Ben Haim, D. Oron, Weizmann Institute of Science (Israel)

7183 IL Surface plasmon-enhanced two-photon fluorescence microscopy for live cell membrane imaging [7183-1 11$]$

R.-Y. He, K.-C. Cho, N.-S. Chang, Y.-D. Su, S.-J. Chen, National Cheng Kung Univ. (Taiwan) 
7183 IN Imaging dental sections with polarization-resolved SHG and time-resolved autofluorescence (Invited Paper) [7183-58]

J. H. Chen, P.-Y. Lin, National Yang-Ming Univ. (United States); S. C. Y. Hsu, National Univ. of Singapore (Singapore); F.-J. Kao, National Yang-Ming Univ. (Taiwan) and National Sun Yat-sen Univ. (Taiwan)

$71831 \mathrm{P} \quad$ Second harmonic generating (SHG) nanoprobes: a new tool for biomedical imaging [7183-60]

P. Pantazis, California Institute of Technology (United States); Y. Pu, D. Psaltis, California Institute of Technology (United States) and École Polytechnique Fédérale de Lausanne (Switzerland); S. Fraser, California Institute of Technology (United States)

7183 IQ Infrared-based least-invasive third and second harmonic generation imaging of ocular tissues [7183-61]

S.-Y. Chen, National Taiwan Univ. (Taiwan); H.-C. Yu, I-J. Wang, National Taiwan Univ. Hospital (Taiwan); C. Sun, National Taiwan Univ. (Taiwan) and Research Ctr. for Applied Sciences (Taiwan)

\section{SESSION 11 HARMONIC GENERATION MICROSCOPY II}

$71831 \mathrm{U}$ Multimodal multiphoton microscopy [7183-65]

F. Légaré, Institut National de la Recherche Scientifique (Canada); C. P. Pfeffer, Harvard

School of Dental Medicine (United States); F. Ganikhanov, West Virginia Univ. (United States)

7183 IV Third and second harmonic generation imaging of human articular cartilage [7183-66] M.-R. Tsai, National Taiwan Univ. (Taiwan); C.-H. Chen, Chang Gung Memorial Hospital at Keelung (Taiwan); C.-K. Sun, National Taiwan Univ. (Taiwan) and Research Ctr. for Applied Sciences (Taiwan)

$7183 \mathrm{iW}$ Structural and molecular conformation of myosin in intact muscle fibers by second harmonic generation [7183-68]

V. Nucciotti, C. Stringari, L. Sacconi, F. Vanzi, M. Linari, G. Piazzesi, V. Lombardi, F. S. Pavone, Univ. of Florence (Italy)

$71831 \mathrm{X}$ Polarization-resolved second-harmonic-generation imaging of photoaged dermal collagen fiber [7183-69]

T. Yasui, Y. Takahashi, T. Araki, Osaka Univ. (Japan)

718312 Imaging of collagen matrix remodeling in three-dimensional space using second harmonic generation and two photon excitation fluorescence [7183-67]

T. Abraham, J. Carthy, B. McManus, St. Paul's Hospital, Univ. of British Columbia (Canada)

POSTER SESSION: STUDENT COMPETITION

718320 Stimulated Raman scattering microscopy for biomedical imaging [7183-43]

W. Min, C. W. Freudiger, S. Lu, Harvard Univ. (United States); C. He, J. X. Kang, Massachusetts General Hospital (United States) and Harvard Medical School (United States); X. S. Xie,

Harvard Univ. (United States) 
718321 Fluorescence lifetime based contrast imaging using variable period excitation pulse trains [7183-71]

M. D. Holton, Swansea Univ. (United Kingdom); O. F. Silvestre, R. J. Errington, P. J. Smith, Cardiff Univ. (United Kingdom); P. Rees, H. D. Summers, Swansea Univ. (United Kingdom)

718323 Improving image formation from the illumination side: linear and non-linear excitation cases (Best Student Poster Award) [7183-73]

E. Ronzitti, Univ. of Genoa (Italy) and Univ. of Milan (Italy); F. Cella, Univ. of Genoa (Italy);

A. Diaspro, Univ. of Genoa (Italy) and Univ. of Milan (Italy)

718324 Non-linear effects and role of scattering in multiphoton imaging of thick biological samples [7183-74]

F. Cella, Z. Lavagnino, Univ. of Genoa (Italy); A. Diaspro, Univ. of Genoa (Italy) and IIT (Italy) and IBF, CNR (Italy)

718325 Mechanical properties of tissue determined by multiphoton microscopy [7183-75]

M. B. Lilledahl, B. Skallerud, C. Davies, Norwegian Univ. of Science and Technology (Norway)

718327 Fluorescence lifetime dynamics of eGFP in protein aggregates with expanded polyQ [7183-78]

V. Ghukasyan, C.-C. Hsu, C.-R. Liu, F.-J. Kao, T.-H. Cheng, National Yang-Ming Univ. (Taiwan)

718328 Lipids distribution imaging of lipid vesicles by multi-focus excitation CARS microscope

[7183-79]

T. Minamikawa, T. Araki, M. Hashimoto, Osaka Univ. (Japan)

$71832 \mathrm{~A}$ Optical detection of concentrations for mixed acid: $\mathrm{HF}$ and $\mathrm{HNO}_{3}$ [7183-82]

G. Kang, K. Kim, Yonsei Univ. (Korea, Republic of)

7183 2B Coherent control in multiphoton fluorescence imaging (Best Student Poster Award) [7183-83]

A. K. De, D. Goswami, Indian Institute of Technology Kanpur (India)

7183 2C High-resolution wavefront correction in multiphoton microscopy [7183-84]

G. Hall, M. Ren, Univ. of Wisconsin, Madison (United States); W. B. Amos, MRC Lab. of Molecular Biology (United Kingdom); K. W. Eliceiri, J. G. White, Univ. of Wisconsin, Madison (United States)

$71832 \mathrm{D}$ Comparison of two-photon imaging depths with $775 \mathrm{~nm}$ excitation and $1300 \mathrm{~nm}$ excitation [7183-86]

D. Kobat, A. Wong, C. B. Schaffer, C. Xu, Cornell Univ. (United States)

$71832 \mathrm{E}$ Localization of protein-protein interactions among three fluorescent proteins in a single living cell: three-color FRET microscopy [7183-87]

Y. Sun, Univ. of Virginia (United States); C. F. Booker, Univ. of Virginia Health System (United

States); R. N. Day, Univ. of Virginia Health System (United States) and Indiana Univ. (United

States); A. Periasamy, Univ. of Virginia (United States)

$71832 \mathrm{~F} \quad$ FRET imaging of multiple focal planes to analyze the organization and conformation of transferrin-receptor in polarized cells [7183-88]

H. Wallrabe, A. Periasamy, Univ. of Virginia (United States); M. Barroso, Albany Medical

College (United States) 
$71832 \mathrm{G}$ Angiotensin II-induced angiotensin II type I receptor lysosomal degradation studied by fluorescence lifetime imaging microscopy [7183-89]

H. Li, P. Yu, Georgetown Univ. Medical Ctr. (United States); R. A. Felder, Univ. of Virginia Health Sciences Ctr. (United States); A. Periasamy, Univ. of Virginia (United States); P. A. Jose, Georgetown Univ. Medical Ctr. (United States)

POSTER SESSION

$71832 \mathrm{~N}$ Fluorescent nanodiamonds for FRET-based monitoring of a single biological nanomotor FoF $_{1}$-ATP synthase [7183-94]

M. Börsch, R. Reuter, G. Balasubramanian, Univ. Stuttgart (Germany); R. Erdmann,

PicoQuant GmbH (Germany); F. Jelezko, J. Wrachtrup, Univ. Stuttgart (Germany)

$71832 \mathrm{Q}$ A setup for combined multiphoton laser scanning microscopic and multi-electrode patch clamp experiments on brain slices [7183-97]

P. J. Helm, T. Reppen, P. Heggelund, Univ. of Oslo (Norway)

$71832 \mathrm{R}$ Transient state microscopy: a new tool for biomolecular imaging [7183-98]

T. Sandén, G. Persson, J. Widengren, Royal Institute of Technology (Sweden)

718325 In vivo and in vitro investigations of retinal fluorophores in age-related macular degeneration by fluorescence lifetime imaging [7183-100]

M. Hammer, S. Quick, Friedrich-Schiller-Univ. Jena (Germany); M. Klemm, Technische Univ. IImenau (Germany); S. Schenke, Friedrich-Schiller-Univ. Jena (Germany); N. Mata, SIRiON Therapeutics Inc. (United States); A. Eitner, D. Schweitzer, Friedrich-Schiller-Univ. Jena (Germany)

$71832 \mathrm{~V}$ Dispersion control considerations for multiphoton and non-linear laser microscopy applications [7183-103]

C. Brideau, P. Stys, Univ. of Calgary (Canada)

718330 Multiphoton microscopy as a diagnostic tool for pathological analysis of sentinel lymph nodes [7183-109]

J. Lemiere, J. Douady, Lab. de Spectrométrie Physique, CNRS, Univ. Joseph Fourier (France);

F. Estève, INSERM, Univ. Joseph Fourier, CEA, CHU (France); D. Salameire, S. Lantuejoul, P. Lorimier, Univ. Joseph Fourier (France) and CHU-Grenoble (France); C. Ricard,

B. van der Sanden, INSERM, Univ. Joseph Fourier, CEA, CHU (France); J.-C. Vial, Lab. de Spectrométrie Physique, CNRS, Univ. Joseph Fourier (France)

718334 Ultra-compact (palm-top size) low-cost maintenance-free ( $>3000 \mathrm{~h}$ ) diode-pumped femtosecond (160 fs) solid state laser source for multiphoton microscopy [7183-114] S. Yamazoe, M. Katou, T. Kasamatsu, Fujifilm Corp. (Japan)

718335 SHIM and TPEM: getting more information from nonlinear excitation [7183-116] P. Bianchini, Univ. of Genoa (Italy); A. Diaspro, Univ. of Genoa (Italy), Italian Institute of Technology (Italy), and IBF, CNR (Italy)

Author Index 
Downloaded From: https://www.spiedigitallibrary.org/conference-proceedings-of-spie on 25 Apr 2023

Terms of Use: https://www.spiedigitallibrary.org/terms-of-use 


\title{
Conference Committee
}

\author{
Symposium Chairs \\ James G. Fujimoto, Massachusetts Institute of Technology (United \\ States) \\ R. Rox Anderson, Wellman Center for Photomedicine, Massachusetts \\ General Hospital (United States), and Harvard School of Medicine \\ (United States)
}

Program Track Chairs

Ammasi Periasamy, University of Virginia (United States)

Daniel L. Farkas, Cedars-Sinai Medical Center (United States)

Conference Chairs

Ammasi Periasamy, University of Virginia (United States)

Peter T. C. So, Massachusetts Institute of Technology (United States)

Program Committee

Wolfgang Becker, Becker \& Hickl GmbH (Germany)

Keith M. Berland, Emory University (United States)

Guy C. Cox, The University of Sydney (Australia)

Alberto Diaspro, Università degli Studi di Genova (Italy)

Chen Y. Dong, National Taiwan University (Taiwan)

Kevin Eliceiri, University of Wisconsin, Madison (United States)

Scott E. Fraser, California Institute of Technology (United States)

Paul M. W. French, Imperial College London (United Kingdom)

Hans C. Gerritsen, Universiteit Utrecht (Netherlands)

Min Gu, Swinburne University of Technology (Australia)

Stefan W. Hell, Max-Planck-Institut für Biophysikalische Chemie (Germany)

Brian A. Herman, The University of Texas Health Science Center at San Antonio (United States)

Satoshi Kawata, Osaka University (Japan)

Karsten König, Saarland Universität (Germany)

Arnd K. Krueger, Spectra-Physics (United States)

Joseph R. Lakowicz, University of Maryland, Baltimore (United States)

Stephen M. McDonald, Coherent, Inc. (United States)

Simon C. Watkins, University of Pittsburgh (United States)

Paul W. Wiseman, McGill University (Canada)

X. Sunney Xie, Harvard University (United States)

Bernhard Zimmermann, Carl Zeiss Jena GmbH (Germany)

Warren R. Zipfel, Cornell University (United States) 
Session Chairs

1 Keynote Session

Ammasi Periasamy, University of Virginia (United States)

2 FLIM/FRET/FCS I

Angelika C. Rueck, Universität Ulm (Germany)

3 FLIM/FRET/FCS ॥

Richard N. Day, University of Virginia Health System (United States)

4 FLIM/FRET/FCS III

Fu-Jen Kao, National Yang-Ming University (Taiwan)

Peter T. C. So, Massachusetts Institute of Technology (United States)

5 CARS Microscopy I

Sunney X. Xie, Harvard University (United States)

6 CARS Microscopy II

Eric O. Potma, University of California, Irvine (United States)

7 CARS Microscopy III

Ji-Xin Cheng, Purdue University (United States)

8 CARS Microscopy IV

Annika M. Enejder, Chalmers Tekniska Högskola (Sweden)

9 Technology Development and Applications

Peter T. C. So, Massachusetts Institute of Technology (United States)

10 Harmonic Generation Microscopy I

Paul J. Capagnola, University of Connecticut Health Center (United States)

11 Harmonic Generation Microscopy II

Karsten König, JenLaboratory GmbH (Germany)

Poster Session: Student Competition

Holly L. Aron, University of California, Berkeley (United States)

Steven S. Vogel, National Institutes of Health (United States)

Christoph U. Biskup, Friedrich-Schiller-Universität Jena (Germany)

Alzbeta Chorvatova, Université de Montréal (Canada) 


\title{
STED microscopy made simple
}

\author{
Andreas Schönle, Lars Kastrup, Katrin Willig, Dominik Wildanger, Benjamin Harke, \\ Brian Rankin, Christian Eggeling, Alexander Egner, Stefan W. Hell \\ Max-Planck-Institut für Biophysikalische Chemie, Germany
}

\begin{abstract}
We present a simple, low-cost STED microscope setups entirely built from standard optical components and based on light sources which are available off the shelve. The devices have been optimized to allow fast alignment through a simple, robust protocol and thus stable operation even by non-expert users. In this configuration, we achieved routine resolutions of down to $70 \mathrm{~nm}$, thus surpassing the diffraction limit 3-fold. This implementation of the STED-principle can therefore serve as a template for researchers seeking to apply non-invasive far-field sub-diffraction imaging or fluorescence fluctuation spectroscopy in nano-sized focal volumes to solve their scientific problems.
\end{abstract}




\title{
Optimization of fluorescence collection in multiphoton microscopy
}

\author{
Joseph Zinter, Michael J. Levene \\ Yale Univ., New Haven, Connecticut
}

\begin{abstract}
Efficient fluorescence collection is critically important to maximize image quality and depth in multiphoton microscopy. Here we present an optimized, large aperture fluorescence collection system for use with the Olympus $20 \times 0.95 \mathrm{NA}$ objective and two Hamamatsu H7422P-40 GaAsP photomultiplier tubes. Using Zemax optical design software to model the fluorescence intensity distribution and collection geometry, we have designed and constructed an optimized, large aperture detector housing assembly, which provides a significant increase in collected fluorescent signal, image quality, and maximum imaging depth.
\end{abstract}

